\title{
Dielectric Relaxation and Hydration Interactions for Protic and Aprotic Ionic Liquids using Time Domain Reflectometry
}

\author{
Shrikant P. Musalea ${ }^{a}$ Ashok C. Kumbharkhane ${ }^{b}$ and Dilip H. Dagade ${ }^{a^{*}}$ \\ a Department of Chemistry, Shivaji University, Kolhapur - 416004, India

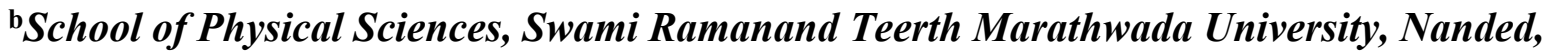 \\ India
}

The supporting information contains relative permittivity and dielectric loss spectra for studied aprotic ionic liquids, [EmimBR, HmimBr and OmimBr], protic ionic liquids, [DEAA, DEAP, DEAB], and their aqueous solutions (DEAA, DEAP, DEAB, EmimBr, BmimBr and $\mathrm{HmimBr}$ ) in the concentration range $\sim 0.02 \mathrm{~mol} \cdot \mathrm{dm}^{-3}$ to $\sim 0.8 \mathrm{~mol} \cdot \mathrm{dm}^{-3}$. The dielectric parameters were obtained by fitting experimental dielectric relaxation spectra using Cole-Cole (CC) model for neat ionic liquids (ILs) and combination of Debye and Cole-Cole model (D+CC) for their aqueous solutions. 
(a)

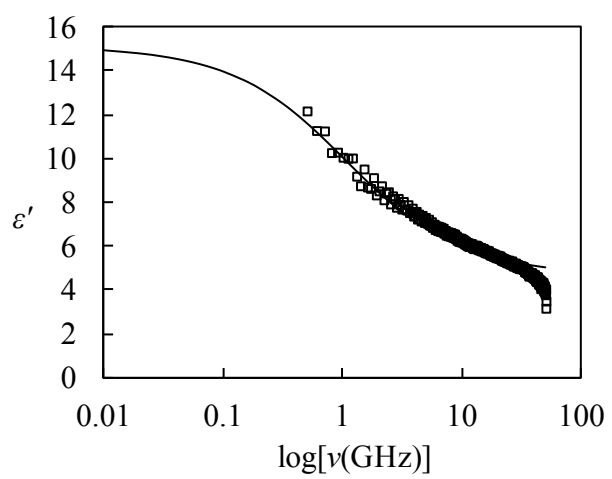

(c)

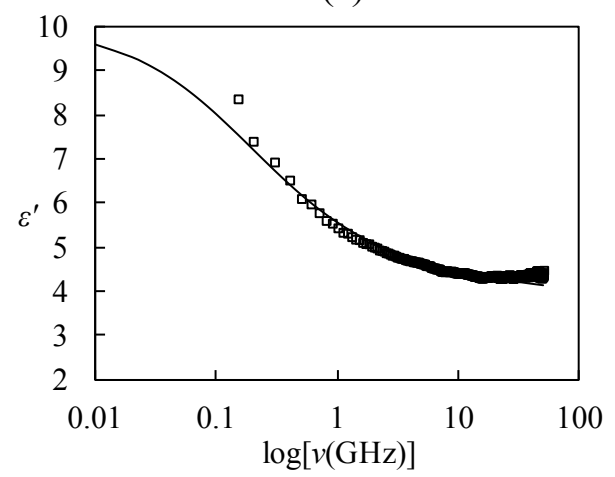

(e)

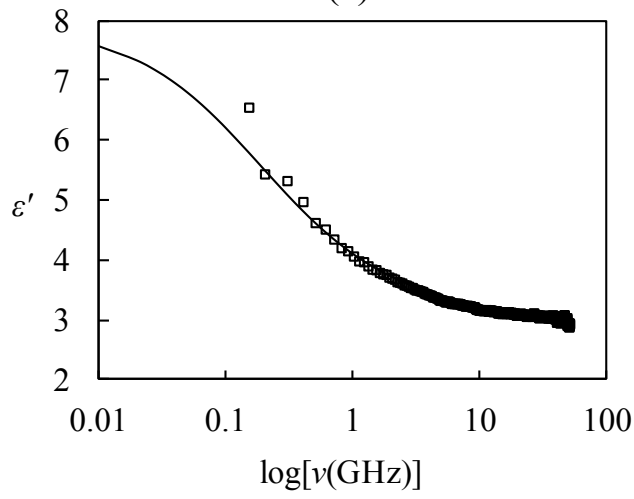

(b)

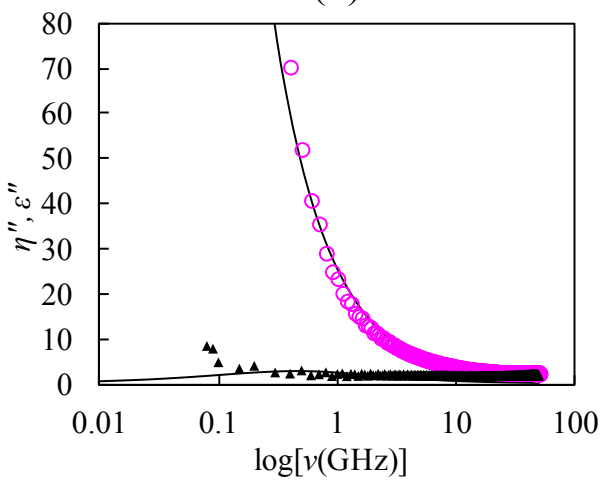

(d)

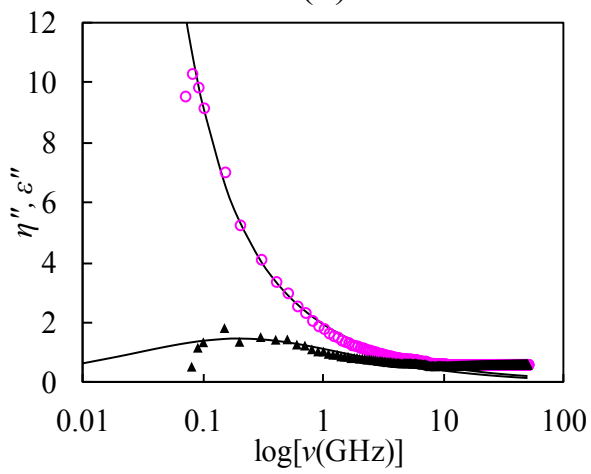

(f)

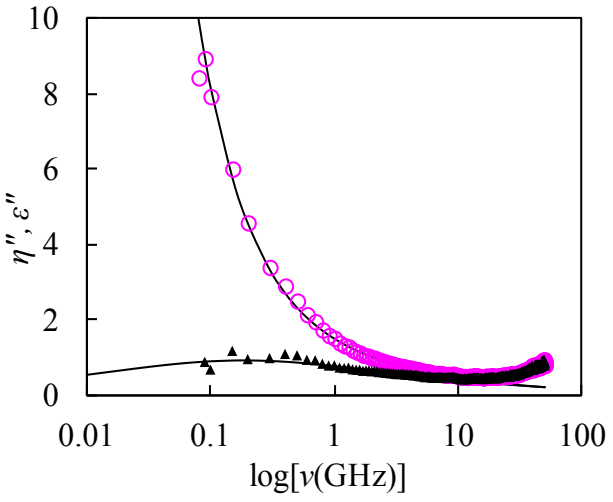

Figure S1 (a), (c) and (e) represents relative permittivity, $\varepsilon^{\prime}(v), \square$; spectra for EmimBr, $\mathrm{HmimBr}$ and OmimBr, Figure (b), (d) and (g) represents total loss, $\eta^{\prime \prime}(v), \circ$; conductivity corrected dielectric loss, $\varepsilon^{\prime \prime}, \mathbf{\Lambda}$; spectra for EmimBr, $\mathrm{HmimBr}$ and $\mathrm{OmimBr}$ at $298.15 \mathrm{~K}$. The symbols represent the experimental data and the black line represent fits obtained with a Cole-Cole dielectric relaxation model. 
(h)

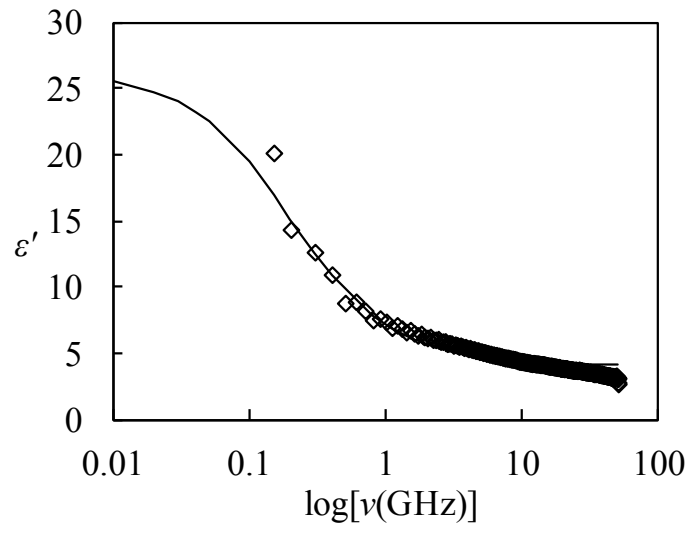

(j)

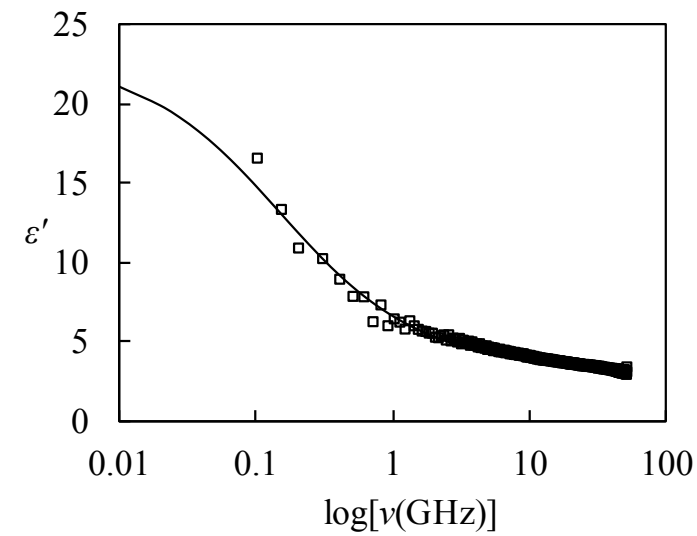

(1)

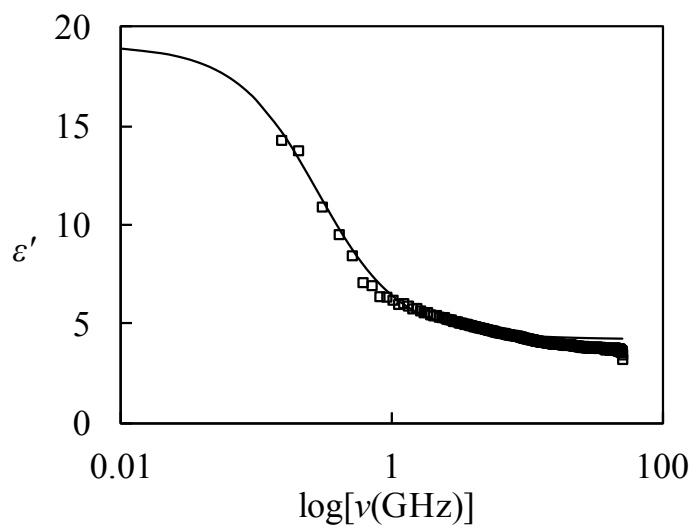

(i)

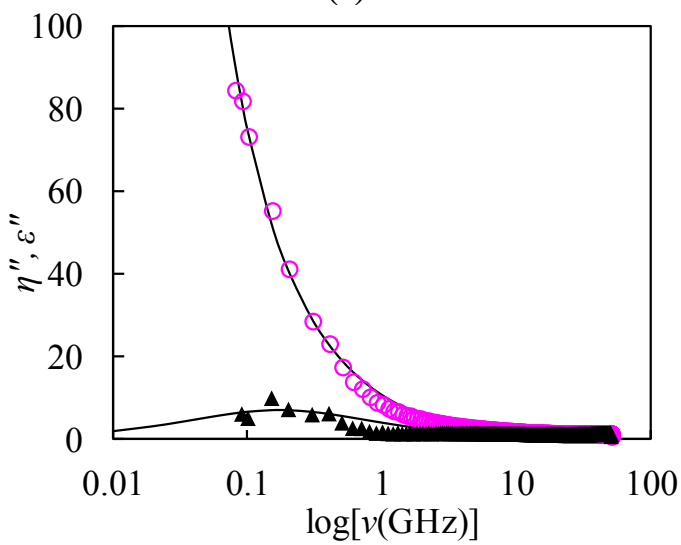

(k)

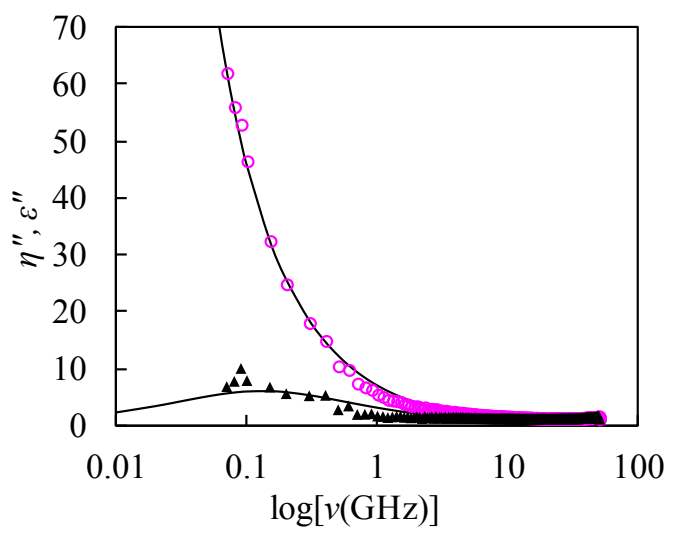

(m)

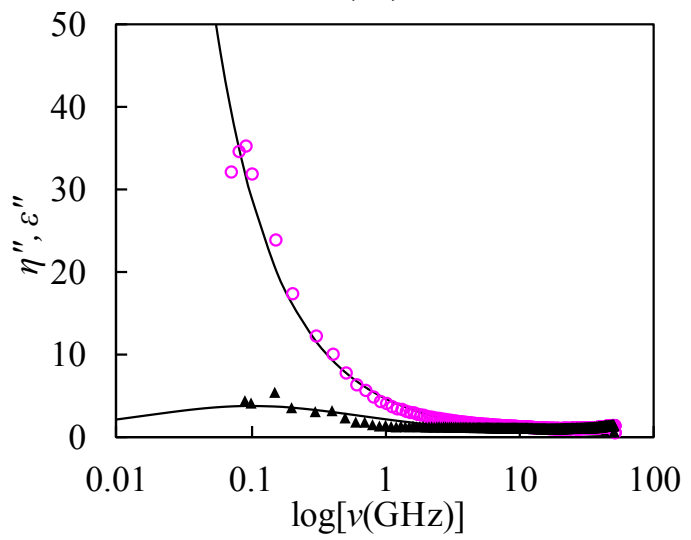

Figure S2 (h), (j) and (l) represents relative permittivity, $\varepsilon^{\prime}(v), \square$; spectra for DEAA, DEAP and DEAB. Figure S2 (i), (k) and (m) represents total loss, $\eta^{\prime \prime}(v), \circ$; conductivity corrected dielectric loss, $\varepsilon^{\prime \prime}, \mathbf{\Delta}$; spectra for DEAA, DEAP and DEAB at $298.15 \mathrm{~K}$. The symbols represent the experimental data and the black line represent fits obtained with a Cole-Cole dielectric relaxation model. 
(a)

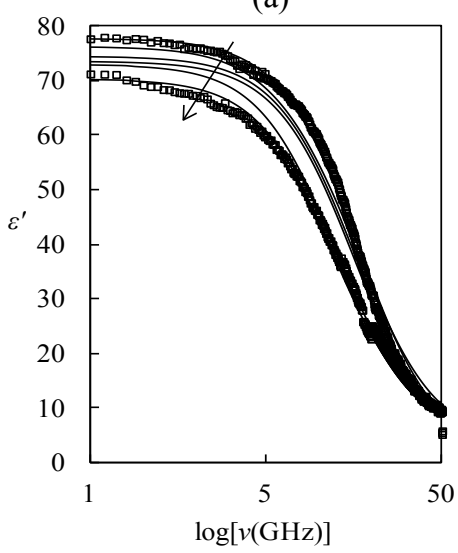

(c)

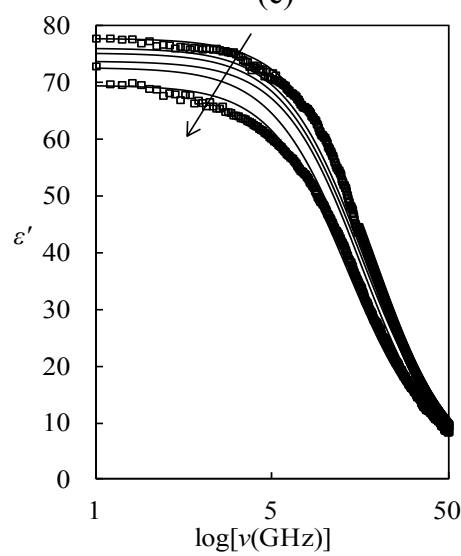

(e)

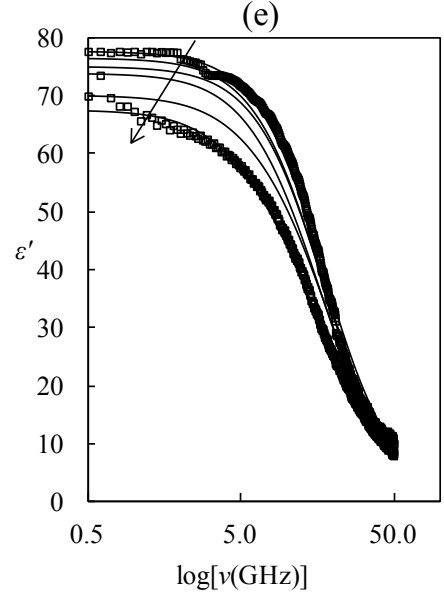

(b)

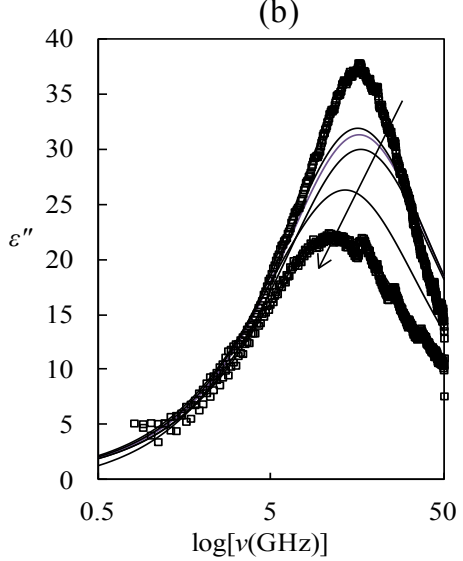

(d)

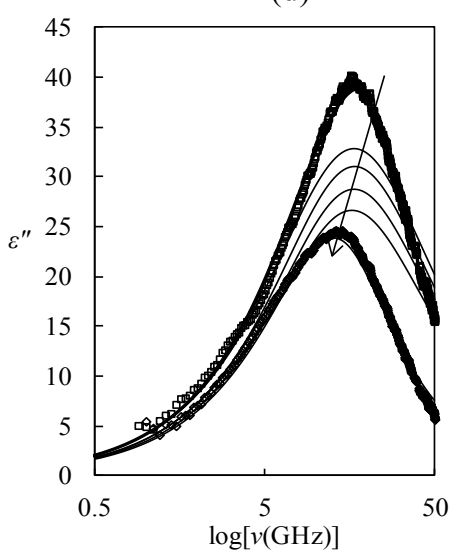

(f)

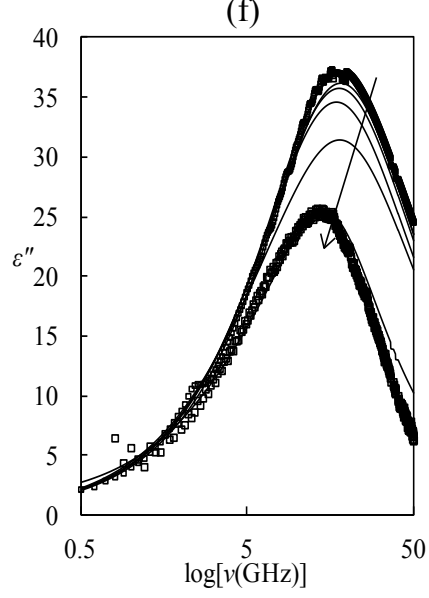

Figure S3. (a) (c) and (e) represents relative permittivity, $\varepsilon^{\prime}(v)$, $\square$; spectra for aqueous solutions of EmimBr, BmimBr and $\mathrm{HmimBr}$ respectively. Figure S3 (b), (d) and (f) represents conductivity corrected dielectric loss, $\varepsilon^{\prime \prime}, \square$; spectra for aqueous solutions of EmimBr, BmimBr and $\mathrm{HmimBr}$ respectively at $298.15 \mathrm{~K}$. Solid black line indicates D+CC model fits. For visual clarity, experimental data (symbols) in spectra are only shown for higher and lower studied concentration. Arrow indicates increasing concentration $\left(\mathrm{mol} \cdot \mathrm{dm}^{-3}\right)$ of APIL. 


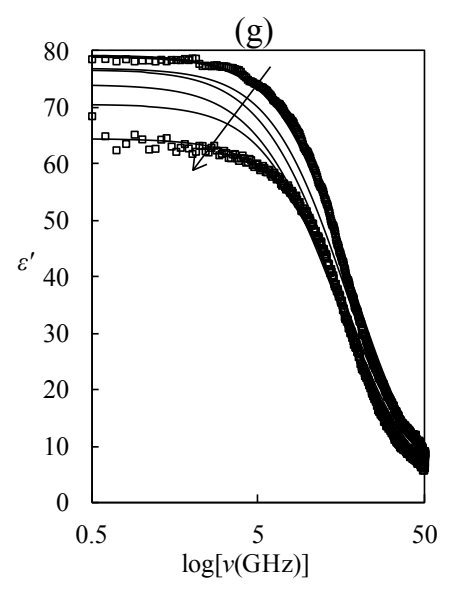

(i)

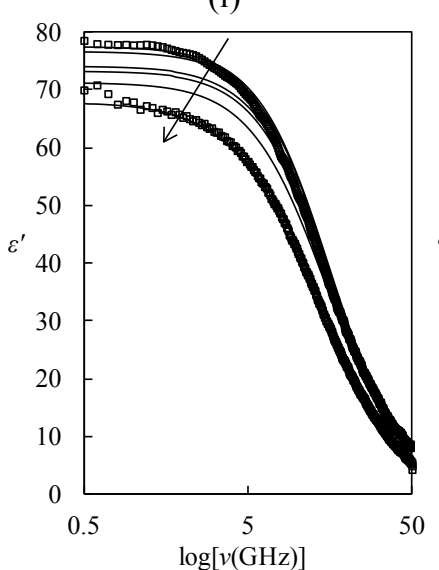

(k)

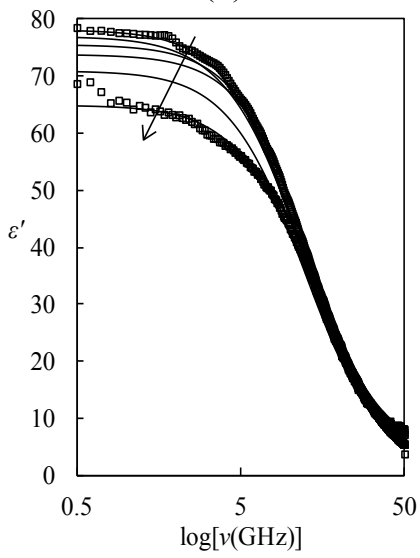

(h)

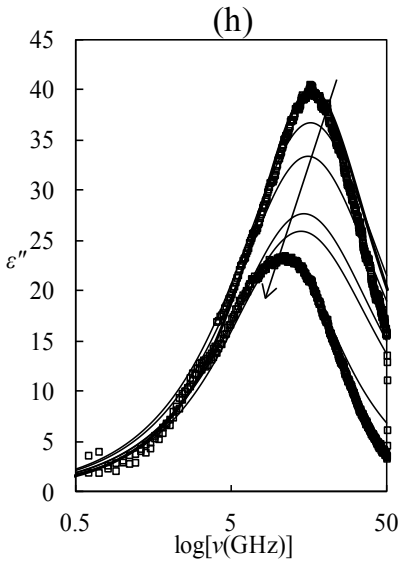

(j)

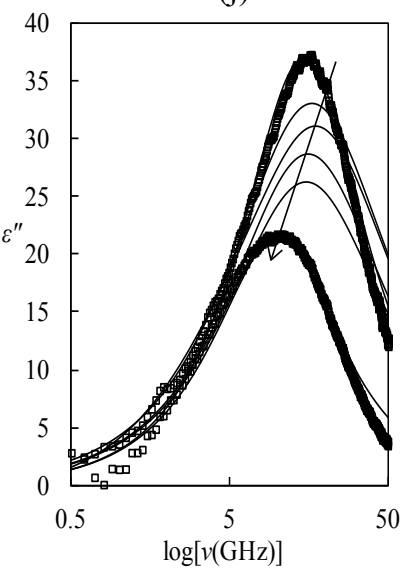

(1)

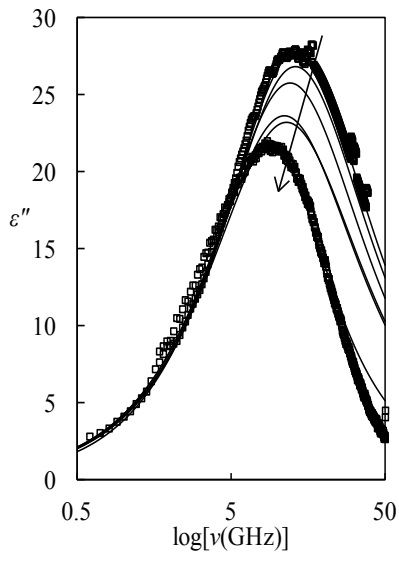

Figure S4. (g) (i) and (k) represents relative permittivity, $\varepsilon^{\prime}(v)$, $\square$; spectra for aqueous solutions of DEAA, DEAP and DEAB respectively. Figure S4 (h), (j) and (l) represents conductivity corrected dielectric loss, $\varepsilon^{\prime \prime}, \square$; spectra for aqueous solutions of DEAA, DEAP and DEAB respectively at $298.15 \mathrm{~K}$. Solid black line indicates D+CC model fits. For visual clarity, experimental data (symbols) in spectra are only shown for higher and lower studied concentration. Arrow indicates increasing concentration $\left(\mathrm{mol} \cdot \mathrm{dm}^{-3}\right)$ of PIL. 